Stearic acid and high molecular weight PEO as matrix for the highly water soluble metoprolol tartrate in continuous twin-screw melt granulation Non Peer-reviewed author version

Monteyne, Tinne; ADRIAENSENS, Peter; Brouckaert, Davinia; Remon, Jean-Paul; Vervaet, Chris \& De Beer, Thomas (2016) Stearic acid and high molecular weight PEO as matrix for the highly water soluble metoprolol tartrate in continuous twin-screw melt granulation. In: INTERNATIONAL JOURNAL OF PHARMACEUTICS, 512(1), p. 158-167.

DOI: 10.1016/j.jpharm.2016.07.035

Handle: http://hdl.handle.net/1942/22756 


\title{
Stearic acid and high molecular weight PEO as matrix for the highly water soluble metoprolol tartrate in continuous twin-screw melt granulation
}

\author{
Tinne Monteyne ${ }^{\mathrm{a}, 1}$, Peter Adriaensens ${ }^{\mathrm{b}}$, Davinia Brouckaert $^{\mathrm{a}}$, Jean-Paul Remon ${ }^{\mathrm{c}}$, Chris Vervaet $^{\mathrm{c}}$, Thomas \\ De Beer ${ }^{\mathrm{a}}$ \\ ${ }^{a}$ Laboratory of Pharmaceutical Process Analytical Technology, Department of Pharmaceutical Analysis, Faculty of \\ Pharmaceutical Sciences, Ghent University, Ottergemsesteenweg 460, 9000 Ghent, Belgium \\ ${ }^{b}$ Laboratory of Applied and Analytical Chemistry, Institute of Material Science, Hasselt University, Campus Diepenbeek, \\ Agoralaan, Building D, Diepenbeek, Belgium \\ ${ }^{c}$ Laboratory of Pharmaceutical Technology, Department of Pharmaceutics, Faculty of Pharmaceutical Sciences, Ghent \\ University, Ottergemsesteenweg 460, 9000 Ghent, Belgium
}

\begin{abstract}
Granules with release-sustaining properties were developed by twin screw hot melt granulation (HMG) using a combination of stearic acid (SA) and high molecular weight polyethylene oxide (PEO) as matrix for a highly water soluble model drug, metoprolol tartrate (MPT), Earlier studies demonstrated that mixing molten $\mathrm{SA}$ and $\mathrm{PEO}$ resulted in hydrogen bond formation between hydroxyl groups of fatty acid molecules and ether groups in $\mathrm{PEO}$ chains. These molecular interactions might be beneficial in order to elevate the sustained release effect of drugs from a $\mathrm{SA} / \mathrm{PEO}$ matrix. This study aims to investigate the continuous twin screw melt granulation technique to study the impact of a SA $/ \mathrm{PEO}$ matrix on the dissolution rate of a highly water soluble drug (MPT). Decreasing the SA $/$ PEO ratio improved the release-sustaining properties of the matrix. The solid state of the granules was characterized using differential scanning calorimetry (DSC), nuclear magnetic resonance (NMR), X-ray diffraction (XRD), Raman spectroscopy, Fourier Transform Infrared (FTIR) and Near Infrared chemical imaging (NIR-CI) in order to understand the dissolution behavior. The results revealed a preferential interaction of the MPT molecules with stearic acid impeding the PEO to form hydrogen bonds with the stearic acid chains. However, this allowed the PEO chains to recrystallize inside the stearic acid matrix after granulation, hence, elevating the release-sustaining characteristics of the formulation.
\end{abstract}

Keywords: Continuous twin-screw melt granulation, Stearic acid, PEO, DSC Vibrational spectroscopy, Solid state NMR Dissolution rate 


\section{Introduction}

Continuous melt granulation is gaining popularity in the pharmaceutical industry due to its multiple advantages. Hot melt granulation (HMG) uses a molten binder to agglomerate the pharmaceutical powder particles. Following particle agglomeration and consolidation, the granules are cooled to room temperature and a solid end product with a granular structure is formed. For this granulation method, the drying step, which is required after wet granulation, is eliminated, hence, the process time and energy requirements are significantly reduced. Since no liquids are used, HMG has the possibility to agglomerate moisture-sensitive materials [? ][? ][? ]. Furthermore, melt granulation can be used to develop high-dose formulations with up to $90 \%$ of active pharmaceutical ingredient (API) [? ][? ].

The type of binder used during melt granulation (and its distribution during processing) is one of the main determinants of the granule quality attributes. Hydrophilic binders, such as low molecular weight polyethylene glycol (PEG) 3000-20,000 or Gelucire 50/13 and 44/14, are typically used to develop immediaterelease systems where the granules quickly disintegrate or dissolve after intake [? ][? ][? ]. In contrast, sustained-release melt granules are prepared via melt granulation using hydrophobic binders, such as fatty acids, microcrystalline wax or hydrogenated castor oil (HCO), which retain the granule's matrix structure in aqueous media [? ][? ][? ]. The binders should have a melting point above $50{ }^{\circ} \mathrm{C}$ to avoid softening of the dosage form during storage and in case of sustained drug delivery systems also to maintain the structural integrity of the matrix at body temperature to avoid excessive drug release after oral delivery [? ].

Previous research has proven that the addition of a hydrophilic polymer to a hydrophobic matrix can modify the drug release from the matrix [? ][?][? ]. However, only a very few studies currently exist in the specific area of using HMG to develop granules containing both hydrophilic and hydrophobic binders. Tiwari et al. firstly prepared melt granules using only $\mathrm{HCO}$ which showed a sustained release where $100 \%$ tramadol was released over 20 hours. The slow release of the drug was attributed to the formation of a uniform coating on the individual drug particles by the hydrophobic polymer during melt granulation hindering penetration of the solvent. Consequently, addition of hydrophilic hydroxypropylmethylcellulose (HPMC) significantly

\footnotetext{
* Corresponding author

Email addresses: Tinne.Monteyne@UGent.be (Tinne Monteyne), Peter.Adriaensens@UHasselt.be (Peter Adriaensens), Davinia.Brouckaert@UGent.be (Davinia Brouckaert), JeanPaul.Remon@UGent.be (Jean-Paul Remon), Chris.Vervaet@UGent.be (Chris Vervaet), Thomas.DeBeer@UGent.be (Thomas De Beer)

${ }^{1}$ Phone number: +32(0)9 2648068

${ }^{2}$ Fax number: +32(0)92648236
} 

44 dissolution profile. was attributed to the presence of both HPMC, which rapidly took up the water leading to swelling, and $\mathrm{HCO}$, which repels the water, resulting in separation of the formulation components. They concluded that the combination of hydrophilic and hydrophobic polymer matrices was not suitable in the development of a controlled release dosage form for water soluble drugs [? ]. However, Vervaeck et al. proposed the use of fatty acids as matrix formers during prilling to control the release of the highly water soluble metoprolol tartrate (MPT). It was concluded that the MPT release could be tailored over a wide range through addition of PEG by varying the PEG concentration and the PEG molecular weight (4000-10,000) [? ]. The use of $\mathrm{PEO}$ in combination with a fatty acid is widely investigated in the field of the phase changing materials $[?][?][?][?][?][?]$. It is discovered that mixing both components in the melt resulted in hydrogen bond formation between hydroxyl groups of fatty acid molecules (proton donor group) and ether groups in PEO chains (proton acceptor group). The occurrence of these molecular interactions might be beneficial in order to elevate the release-sustaining properties of a drug from a SA/PEO matrix. Therefore, this research aims to investigate the continuous twin screw melt granulation technique to study the use of a SA PEO matrix on the dissolution rate of a highly water soluble drug (MPT). The solid state of the granules, was characterized using DSC, NMR, XRD, Raman spectroscopy, FTIR and NIR-CI in order to understand the

\section{Materials and methods}

46 2.1. Materials

SA with a C18 purity of $98.7 \%$ melting temperature $\left(T_{m}\right)=69^{\circ} \mathrm{C}$ ) was purchased from Mosselman (Ghlin,

56 given in table 1 
Table 1: Experimental powder mixtures in varying SAPEO and MPT content $(\% \mathrm{w} / \mathrm{w})$.

\begin{tabular}{|c|c|c|c|}
\hline Blend & SA & PEO & MPT \\
\hline \hline F1 & 90 & 10 & 0 \\
F2 & 80 & 20 & 0 \\
F3 & 75 & 25 & 0 \\
F4 & 70 & 30 & 0 \\
F5 & 60 & 40 & 0 \\
\hline M1 & 85.5 & 9.5 & 5 \\
M2 & 76 & 19 & 5 \\
M3 & 71.25 & 23.75 & 5 \\
M4 & 66.5 & 28.5 & 5 \\
M5 & 57 & 38 & 5 \\
\hline
\end{tabular}

\subsection{Twin-screw melt granulation}

Melt granulation was performed using a co-rotating intermeshing twin-screw granulator (Prism Eurolab 16) (Thermo Fisher Scientific, Karlsruhe, Germany) with a barrel length of $25 L / D$, where $L$ is the axial screw length of the machine and $D$ is the inner bore diameter. The screw design was identical for all experiments containing one kneading zone located in the fifth segment and consisting of 6 kneading discs positioned at a $60^{\circ}$ stagger angle in reversed direction. The premixed samples (Table 1 ) were fed into the granulator using a DD Flexwall 18 gravimetric feeder (Brabender Technologie, Germany), which was set in the gravimetric feeding mode. Throughput and screw speed were kept constant at $0.3 \mathrm{~kg} / \mathrm{h}$ and $150 \mathrm{rpm}$, respectively. The barrel was divided into 6 barrel zones. Barrel temperature from segment 2 to 5 was kept constant at $60^{\circ} \mathrm{C}$. Segment 6 , which is located at the end of the barrel, had a lower temperature of $40{ }^{\circ} \mathrm{C}$ during all runs in order to cool down the granules and hence avoiding them to stick together when leaving the granulator. Granule samples were collected after melt granulation of each mixture (Table 1). Afterwards, samples were stored at room temperature.

\subsection{In vitro dissolution}

In vitro dissolution was performed using USP dissolution apparatus 1 (baskets). The equipment consisted of a VK 7010 dissolution system coupled with a VK 8000 automatic sampling station (Vankel, New Jersey, USA). An amount of granules, corresponding to $30 \mathrm{mg}$ MPT, was inserted into the baskets. Basket rotational speed was set at $100 \mathrm{rpm}$ and the temperature of the dissolution medium was maintained at $37^{\circ} \mathrm{C}$. Samples of $5 \mathrm{~mL}$ were withdrawn after $0.5,1,2,4,6,8,12,16,20$ and 24 hours and spectrophotometrically analyzed at $222 \mathrm{~nm}$ using a double beam spectrophotometer (UV-1650PC, Shimadzu, Antwerp, Belgium). A validated calibration model was used for quantification of the MPT. Each experiment was performed in triplicate. 
2.4. Solid state NMR

Carbon-13 solid-state CP/MAS NMR spectra were acquired on an Agilent VNMRS DirectDrive $400 \mathrm{MHz}$ spectrometer (9.4 T wide bore magnet) equipped with a T3HX $3.2 \mathrm{~mm}$ probe dedicated for small sample volumes and high decoupling powers. Magic angle spinning (MAS) was performed at $7 \mathrm{kHz}$ in ceramic rotors of $3.2 \mathrm{~mm}(22 \mu \mathrm{l})$. The aromatic signal of hexamethylbenzene was used to determine the Hartmann-Hahn condition $\left(\omega_{1 H}=\gamma_{H} \cdot B_{1 H}=\gamma_{C} \cdot B_{1 C}=\omega_{1 C}\right)$ for cross-polarization $(\mathrm{CP})$, and to calibrate the carbon chemical shift scale (132.1 ppm).

Acquisition parameters used for the carbon spectra and $\mathrm{T} 1 \mathrm{H}$ measurements were: a spectral width of $50 \mathrm{kHz}$, a $90^{\circ}$ pulse length of $2.5 \mu \mathrm{s}$, a spin-lock field for $\mathrm{CP}$ of $100 \mathrm{kHz}$, a contact time for CP of $0.5 \mathrm{~ms}$, an acquisition time of $30 \mathrm{~ms}$ and a recycle delay time of $60 \mathrm{~s}$. High power proton dipolar decoupling was set to $100 \mathrm{kHz}$ during the acquisition time.

The $\mathrm{T} 1 \mathrm{H}$ relaxation decay times were measured, via the carbon signals, by the Inversion Recovery method. The signal intensities were analyzed mono-exponentially as a function of the variable inversion time $t$ according to:

$$
I(t)=I_{o} \cdot\left(1-2 \cdot \exp ^{\left(-t / T_{1 H}\right)}\right)+c t e
$$

The experimental $\mathrm{T} 1 \mathrm{H}$ relaxation data were analyzed by a non-linear least-squares fit (LevenbergMarquardt algorithm).

\subsection{Differential scanning calorimetry}

DSC was used to measure the onset $T_{m}$ and melt enthalpy $(\Delta H)$ of the pure compounds, physical mixtures and the corresponding granule samples obtained after melt granulation of the blends from table 1 . A DSC Q2000 calorimeter (TA Instruments, Zellik, Belgium) was used. Samples ( $\pm 5 \mathrm{mg}$ ) were hermetically sealed in aluminium pans. A heat-cool-heat method with a linear heating rate of $10^{\circ} \mathrm{C} / \mathrm{min}$ was applied for the physical mixtures, whereas only one heating was performed for the granulated samples (also heating rate of $10^{\circ} \mathrm{C} / \mathrm{min}$ ). Dry nitrogen was used as a purge gas through the DSC at a flow rate of $50 \mathrm{~mL} / \mathrm{min}$. Analysis was performed in triplicate. 


\subsection{Fourier Transform Infra-red}

Attenuated Total Reflection (ATR)FTIR spectroscopy was performed on pure substances, physical mixtures and corresponding granules in order to identify molecular interactions formed during continuous twin screw melt granulation. Spectra were recorded using a Nicolet iS5 ATR FTIR (Thermo Fisher Scientific). Each spectrum was collected in the range $4000-550 \mathrm{~cm}^{-1}$ with a resolution of $8 \mathrm{~cm}^{-1}$ and averaged over 32 scans. All spectra were Standard Normal Variate (SNV) -preprocessed to eliminate the physical variation and environmental noise in order to enhance the contribution of the chemical composition.

\subsection{Raman}

Raman spectra were collected with a Raman Rxn1 spectrometer (Kaiser Optical Systems, Ann Arbor, MI, USA), equipped with an air-cooled CCD detector. The laser wavelength was the $785 \mathrm{~nm}$ line from a $785 \mathrm{~nm}$ Invictus NIR diode laser. All spectra were recorded over the $100-1800 \mathrm{~cm}^{-1}$ range with a resolution of $4 \mathrm{~cm}^{-1}$, an exposure time of $10 \mathrm{~s}$ and averaged over 3 scans. A laser power of $400 \mathrm{~mW}$ was used. Data analysis was performed using SIMCA P+(Version 14, Umetrics, Umea, Sweden). All spectra were SNV preprocessed to remove the influence of physical properties and environmental noise in order to enhance the contribution of the chemical composition. Raman spectroscopy was performed on the pure compounds, the physical mixtures, and the corresponding granules.

\subsection{X-ray diffraction}

Crystallinity was analyzed using X-ray diffraction on the pure compounds, the physical mixtures, and the corresponding granules. X-ray diffraction was performed with a D5000 Cu K $\alpha$ diffractor $(\mathrm{k}=1.54 \mathrm{~A})$ (Siemens, Karlsruhe, Germany) with a voltage of $40 \mathrm{mV}$ in the angular range of $10^{\circ}<2 \mathrm{~h}<60^{\circ}$ using a step scan mode ( step size $=0.02^{\circ}$, counting time $\left.=1 \mathrm{~s} / \mathrm{step}\right)$.

\subsection{NIR chemical imaging}

A square of circa $25 \mathrm{~cm}^{2}$ was covered with granules collected during each HMG experiment and was analyzed using NIR-CI in order to visualize the matrix material on the surface of the granules. The Near Infrared (NIR) spectral images of the granules were collected using a Pushbroom line-scanning hyperspectral camera (VLNIR, Specim Ltd., Oulu, Finland). The granules were placed on a black background. The Imspector N17E spectrograph scans a row of 320 spatial pixels at a time (spatial resolution was $32 \mu \mathrm{m})$, and disperses the incoming light from each pixel in the spectral range $900-1700 \mathrm{~nm}$ onto one column of the 320 x 256-pixel Indium Gallium Arsenide (InGaAs)-detector (12-bit readout, thermoelectrically cooled). The 
granules were illuminated with a set of three halogen lamps in a 45-0 configuration (Specim Ltd., Oulu, Finland). Hence, the set of lamps shine light onto the sample surface at an angle of $45^{\circ}$ and the camera looks directly down towards the sample (Figure 1). For each granule sample, a row of granules was measured. From the total NIR-CI-image, a square (150x150pixels) was selected. The spectral data of this square were preprocessed by a combination of SNV and second derivative correction. The mean spectrum of each square was calculated to visually inspect the spectral differences. Multivariate curve resolution (MCR) analysis was applied to the preprocessed squares in order to estimate the relative concentration of each component on the surface of the granules.

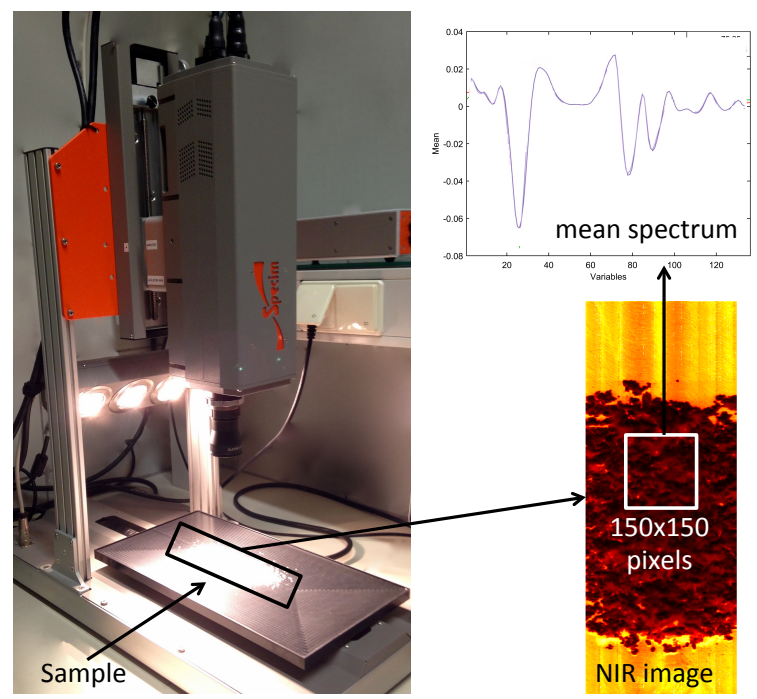

Figure 1: NIR-CI set-up and visualization data analysis to reach a mean spectrum of a NIR-image.

MCR aims to obtain a clear description of the contribution of each material component in the formulation from the overall measured variation in the data matrix (D). Hence, all collected spectra in the image are considered as the result of the additive contribution of all pure components involved in the formulation. Therefore, MCR decomposes D into the contributions linked to each of the pure components in the system:

$$
D=C \cdot S^{T}+E
$$

with $C$ and $S$ representing the concentration profile and spectra, respectively. $E$ is the error matrix. The working procedure started with the initial estimation of $\mathrm{C}$ and $\mathrm{S}$ and worked by optimizing iteratively the concentration and response profiles using the available information about the system. This procedure is named the iterative alternating least squares resolution method (MCR-ALS). The introduction of this 
information was carried out through the implementation of constraints. Constraints are mathematical or chemical properties systematically fulfilled by the whole system or by some of its pure contributions. The only constraint used for this study was the default assumption of non-negativity in the row mode; i.e. negative elements are not allowed in the concentration profile $\mathrm{C}$. The initial estimates provided to the system were the median spectra of the NIR-CI data of pure SA and PEO, preprocessed in an identical way as the granule images. The MCR analysis was performed using the MCR-ALS GUI created by Jaumot et al., running on MATLAB (version R2015a, The Mathworks Inc., USA) [? ].

\section{Results}

In vitro dissolution was executed to examine the influence of $\mathrm{PEO}$ on the release rate of $\mathrm{MPT}$ from a stearic acid matrix developed during continuous hot melt granulation. Figure 2 represents the dissolution profiles in water for granules containing 5\% MPT in a stearic acid matrix with increasing PEOlM content. At low PEO fractions (blend M1), 100\% of the drug was released in 4h. However, a large burst effect was observed, releasing already $77 \%$ MPT after $0.5 \mathrm{~h}$. The sustained-release properties did improve at increasing PEO concentrations where only $90 \% \mathrm{MPT}$ was released after $24 \mathrm{~h}$ for granules containing $38 \%$ PEO. Additionally, the burst effect decreased with a release of only $28 \%$ MPT in 0.5 h (blend M5).

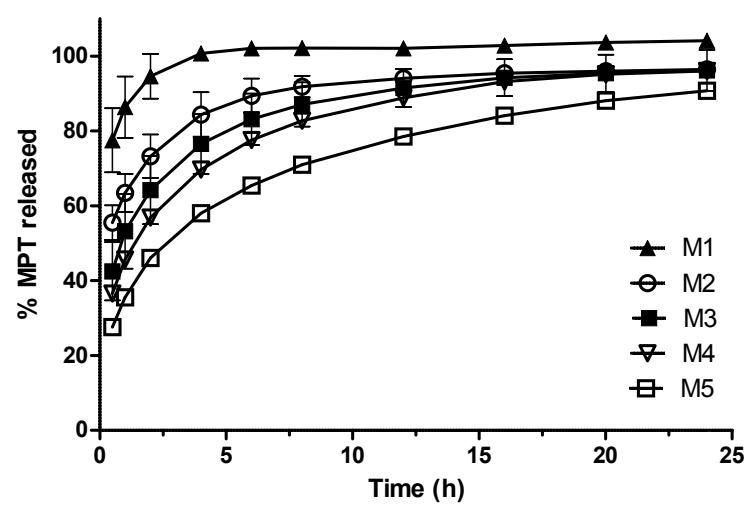

Figure 2: Release of $5 \% \mathrm{MPT}$ from a $\mathrm{SA}$ PEO matrix with varying ratio (blends M1-M5).

The influence of PEG4000-10,000 on the dissolution rate of MPT from a behenic acid matrix was already studied earlier for a prilling process by Vervaeck et al. [? ]. An accelerated drug release was observed at increasing PEG concentrations. Complete drug release (10\% MPT) was observed after 24h when 5\% PEG 
was added to the fatty acid matrix whereas complete drug release was already obtained after $12 \mathrm{~h}$ when using 10\% PEG. Furthermore, addition of PEG to the behenic acid matrix induced a burst release which increased when prilling was executed using higher PEG fractions. Performing melt granulation using decreasing $\mathrm{SA} / \mathrm{PEO} 1 \mathrm{M}$ ratios had an opposite effect on the burst effect and release rate of MPT from a fatty acid matrix compared to prilling using PEG 4000-10,000. An increasing PEO1M fraction caused a more sustained release of MPT with a lower burst effect during melt granulation whereas the use of higher PEG 4000-10,000 fractions during prilling stimulated the drug release with a higher burst effect. During prilling, the PEG chains possibly generated an extended channel system in the prills, destroying the matrix effect of behenic acid. Hence, the release of MPT was accelerated. Since addition of PEO1M had a release-sustaining effect, the extended channel system can not be the reason for the delayed dissolution rate of MPT from the granules. Low (PEG4000-10,000) and high (PEO 1,000,000) molecular weight PEG/PEO behave different after hydration. Low molecular PEG's dissolve into the medium, whereas high molecular weight PEO starts swelling, forming a gel-structure. Therefore, solid state characterization of the granules was performed to understand the dissolution behavior of MPT from a SA PEOlM matrix.

Earlier research discovered that mixing $\mathrm{SA}$ and $\mathrm{PEO}$ in the melt resulted in hydrogen bond formation between hydroxyl groups of fatty acid molecules and ether groups in PEO chains [? ][? ]. The occurrence of these molecular interactions might be beneficial in order to enhance the sustained release effect of a drug from a SA PEO matrix. Therefore, drug-free granules (blends F1-F5) were analyzed in order to study if these interactions were also present after melt granulation and whether they were maintained after addition of MPT (blends M1-M5).

The FTIR spectra demonstrated that interaction did occur between PEO and SA after melt granulation of the drug-free blends (F1-F5). The most obvious changes in comparison with the physical mixtures could be observed in the peaks due to the PEO molecules. Peak $842 \mathrm{~cm}^{-1}$, which is attributed to the C-O and C-C stretching vibration and $\mathrm{CH}_{2}$ rocking vibration of the $\mathrm{PEO}$ ethylene groups shifted to higher wavenumbers in comparison with the physical mixture (Figure 3). The shift was not visible for blends of ratio SA/PEO $60 / 40$ and ranged from $1 \mathrm{~cm}^{-1}$ for granules of ratio SA/PEO $75 / 25$ to $2.6 \mathrm{~cm}^{-1}$ after granulation of blend $90 / 10$. Peak $960 \mathrm{~cm}^{-1}$ disappeared and peak $1060 \mathrm{~cm}^{-1}$ became broader and shifted $1 \mathrm{~cm}^{-1}$ to higher wavenumbers in comparison with the physical mixtures. Peaks $960 \mathrm{~cm}^{-1}$ and $1060 \mathrm{~cm}^{-1}$ were due to the C-O-C stretching vibration of $\mathrm{PEO}$. These shifts revealed an interaction with the ether oxygens in the PEO chains which 
counteracts the $\mathrm{C}-\mathrm{O}-\mathrm{C}$ stretch causing vibration at higher wavenumbers. At the same time, the $\mathrm{CH}_{2}$ wagging and C-C stretching vibrations of $\mathrm{PEO}$, appearing at respectively $1342 \mathrm{~cm}^{-1}$ and $1360 \mathrm{~cm}^{-1}$, decreased in intensity and broadened which can be seen in figure 3. The latter effect can be assigned to the lower bond strength between C-C due to the interaction at the C-O-C band. Spectroscopic deviations were also seen for stearic acid. The $\mathrm{CH}_{2}$ and $\mathrm{CH}_{3}$ rocking vibrations in the range $720-888 \mathrm{~cm}^{-1}$ broadened after granulation. Additionally, the C-O stretching vibrations of the acidic functional group positioned at 1278 and $1296 \mathrm{~cm}^{-1}$ broadened and the latter peak shifted $1.2 \mathrm{~cm}^{-1}$ to higher wavenumbers. Furthermore, the peak ratio of both peaks changed since peak $1278 \mathrm{~cm}^{-1}$ became less intense. Pielichowska assigned the $1278 \mathrm{~cm}^{-1}$ peak to the C-O stretching vibration in a system with hydrogen bonds [? ]. This means that less stearic acid dimers were formed after granulation compared to the physical mixtures. In the physical mixtures, small bands could be observed at 1540 and $1577 \mathrm{~cm}^{-1}$ due to the carboxylate peak. However, the bands could not be detected anymore after granulation. A shift of $2 \mathrm{~cm}^{-1}$ to higher wavenumbers could be observed for the C-O stretching and $\mathrm{C}-\mathrm{O}-\mathrm{H}$ in plane bending at $1430 \mathrm{~cm}^{-1}$ for blend $60 / 40$ which decreased to $1.3 \mathrm{~cm}^{-1}$ for blend $75 / 25$ and was disappeared for blend $90 / 10$. The $\mathrm{C}=\mathrm{O}$ stretch of the $\mathrm{C}-\mathrm{O}-\mathrm{O}-\mathrm{H}$ function $\left(1699 \mathrm{~cm}^{-1}\right) \mathrm{be}^{-}$ came broader after granulation and was most pronounced as the $\mathrm{PEO}$ concentration increased. Finally, the $\mathrm{C}-\mathrm{H}$ stretching vibrations of the $\mathrm{CH}_{2}$ at $2914 \mathrm{~cm}^{-1}$ became broadened. These spectral observations clearly showed that hydrogen bond formation did occur between the acidic group of stearic acid and the C-O-C of PEO after continuous melt granulation. Since peak $1699 \mathrm{~cm}^{-1}$ broadened, most likely also interaction occurred between the $\mathrm{C}=\mathrm{O}$ of stearic acid and the $\mathrm{O}-\mathrm{H}$ end groups of $\mathrm{PEO}$, which are not recognizable in the FTIR spectra.

When MPT was added to the blend, a SA.MPT interaction did occur making the entire PEO molecules less visible. Characteristic FTIR bands for MPT were found in the physical mixtures at 604, 822, 1250, 1382 and $1580 \mathrm{~cm}^{-1}$, representing the out-of-plane $\mathrm{O}-\mathrm{H}$ vibration of alcohol, out-of-plane $\mathrm{O}-\mathrm{H}$ vibration of the carbonyl group, the $\mathrm{O}-\mathrm{H}$ deformation, the aromatic ring stretching and the $\mathrm{CO}_{2}$ antisymmetric stretching vibration of the MPT. In the granules, the bands at 822 and $1580 \mathrm{~cm}^{-1}$ had broadened and the bands at 1250 and $1382 \mathrm{~cm}^{-1}$ disappeared as showed in figure 4. These changes indicated a loss in crystallinity and the existence of amorphous MPT fractions. Simultaneously, the bands due to the PEO vibrations became less pronounced after granulation and the spectrum shifted in the direction of the spectrum of pure stearic acid. This was most remarkable for peaks 842,1147 and $1347 \mathrm{~cm}^{-1}$ which were almost undetectable after granulation. It was also noticeable for peak $1099 \mathrm{~cm}^{-1}$ which was deformed to the shape of the stearic acid 

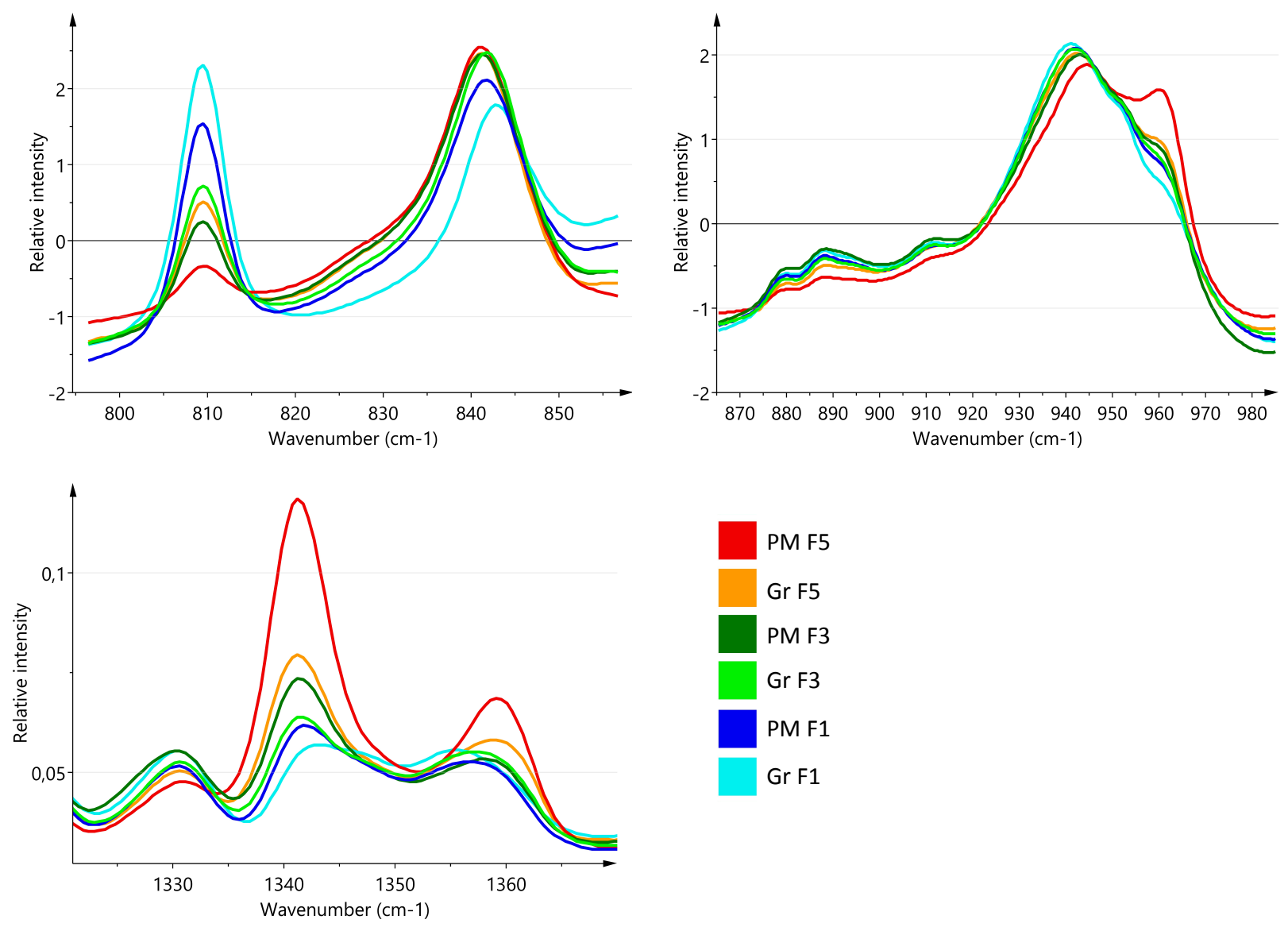

Figure 3: FTIR signals of drug-free physical mixtures (F1, F3 and F5) and corresponding granules for regions $800-860 \mathrm{~cm}^{-1}, 865-980 \mathrm{~cm}^{-1}$ and $1320-1370 \mathrm{~cm}^{-1}$.

peak of $1103 \mathrm{~cm}^{-1}$. However, at elevated PEO concentrations, the PEO became more visible which was the case for blend SA PEO $/$ MPT $57 / 38 / 5$. The reason for the less pronounced PEO vibrations will be discussed later.

The formation of hydrogen bonds and Van der Waals interactions between stearic acid and the entire MPT molecule was detected earlier by Vervaeck et al., where the representative Raman and FTIR peaks for MPT had broadened or disappeared after prilling [? ]. Vervaeck and coworkers suggested that an amorphous MPT fraction was distributed as separate phases throughout the entire fatty acid matrix. However, their XRD results revealed diffraction peaks of MPT demonstrating that the crystalline state of MPT was at least partially maintained after the prilling process. Apparently, during granulation of the SAPEO MPT blend, a competition between $\mathrm{PEO}$ and MPT for the interaction with stearic acid did occur. The FTIR spectra revealed interaction of the fatty acid with MPT whereas interaction with PEO was impeded and as 

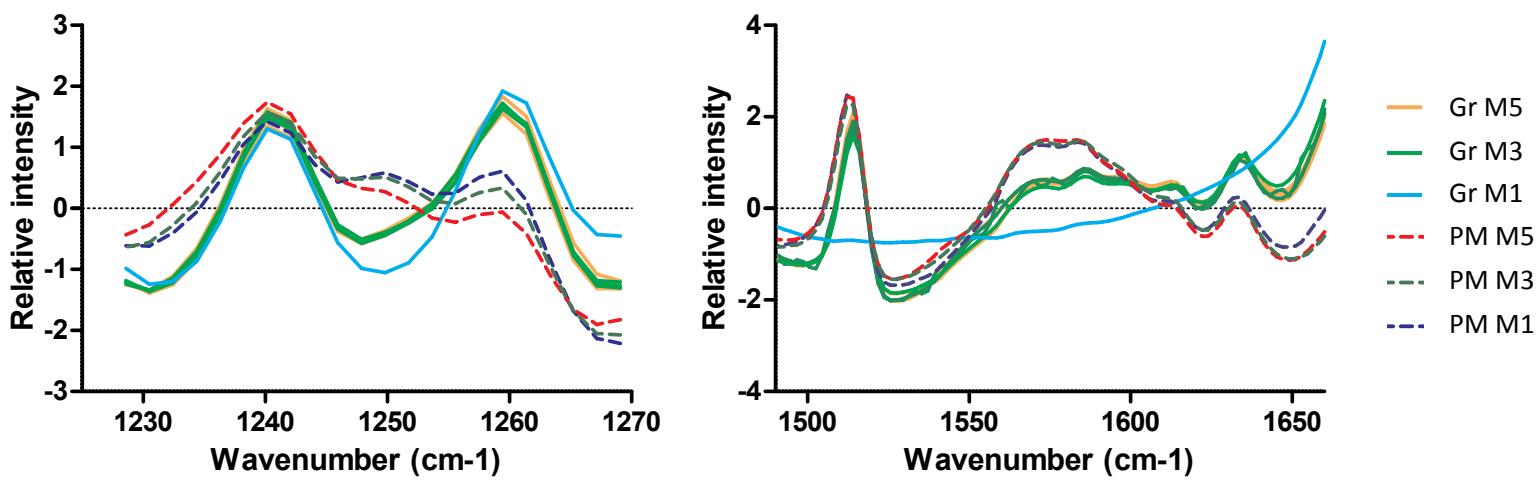

Figure 4: FTIR signals of MPT containing physical mixtures (M1, M3 and M5) and corresponding granules for region (left) $1230-1270 \mathrm{~cm}^{-1}$ and (right) $1500-1650 \mathrm{~cm}^{-1}$.

a result, the $\mathrm{PEO}$ molecules became less visible. Since no interactions between $\mathrm{SA}$ and $\mathrm{PEO}$ occurred, these materials behave as two separate phases allowing stearic acid to partially cover the $\mathrm{PEO}$ molecules, hence making them less visible for spectroscopic measurements.

In order to investigate the components on the granule surface, NIR-CI was executed on drug-free (F1, F3 and F5) and MPT containing granules (M1, M3 and M5). The mean spectra of the drug-free and MPT containing granules demonstrate the spectral differences and are shown in figure 5 . The spectra of the MPT containing granules (green) are more dominated by the pure stearic acid spectrum in comparison with the drug-free granules (red). Hence, an increased appearance of stearic acid on the surface of the MPT containing granules can be assumed. $\mathrm{PEO}$, on the other hand, was clearly visible on the surface of the drug-free granules whereas the visibility decreased after addition of MPT. Furthermore, MCR was applied on the preprocessed spectral data in order to obtain the relative contribution of both components, $\mathrm{SA}$ and $\mathrm{PEO}$, on the surface of the granules. Therefore, the contribution spectra obtained after MCR analysis had to correspond with the spectra of the pure components, which was the case. The obtained concentration of SA and PEO is shown in figure 6. These results revealed that stearic acid was more present on the surface of the MPT containing granules compared to the drugfree granules with the same SA PEO ratio. This confirmed the aforementioned hypotheses that $\mathrm{PEO}$ was less visible during FTIR experiments because $\mathrm{PEO}$ got partially covered with $\mathrm{SA}$ when $\mathrm{MPT}$ was added to the blend. Hence, the lower intensity of the PEO peaks could be explained.

Raman results confirmed the interaction between stearic acid and MPT Since the peaks throughout the complete spectrum of MPT disappeared or reduced in intensity, it can be concluded that the entire MPT 

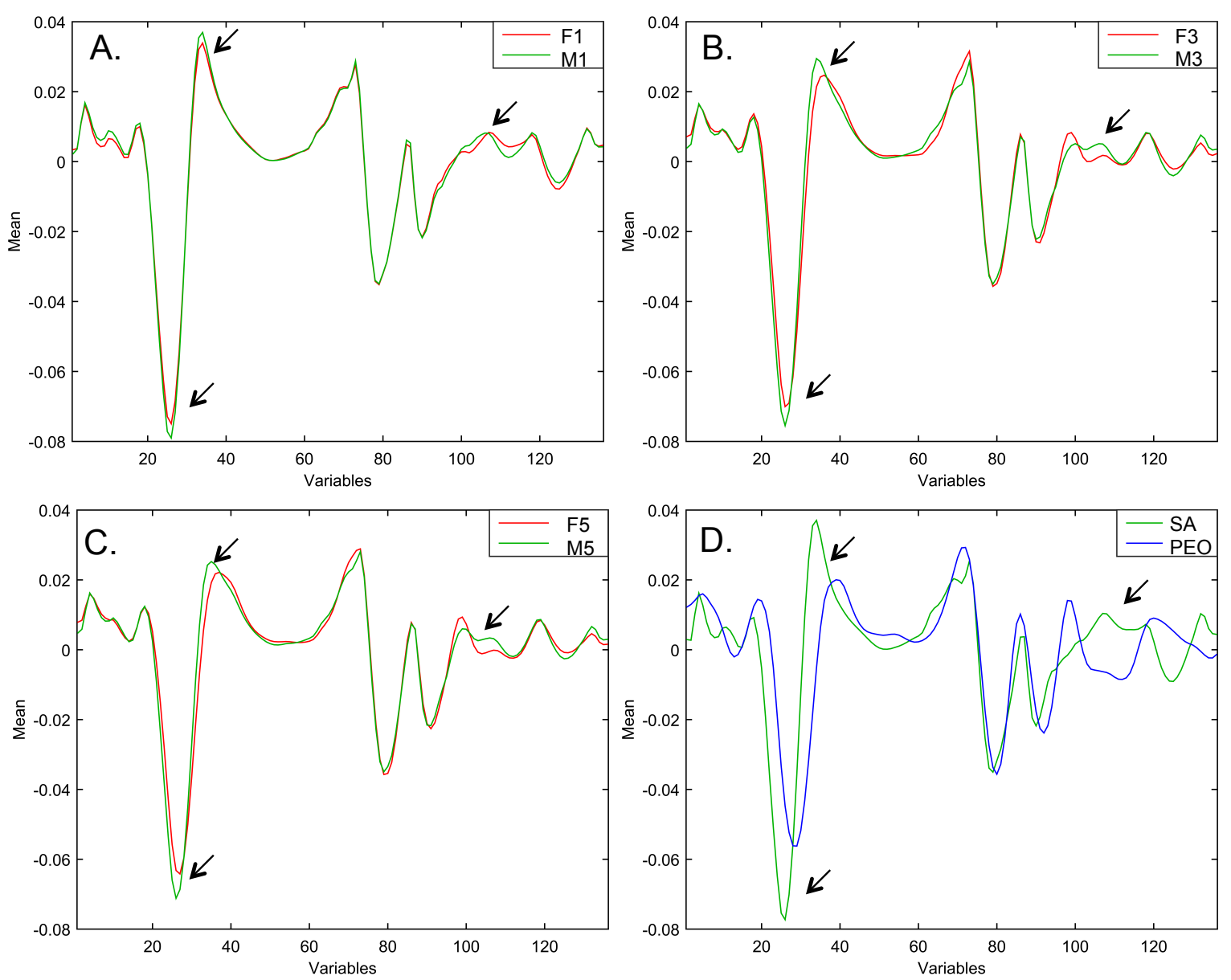

Figure 5: Mean spectra obtained through imaging the surface of the granules of blends F1, F3 and F5 (red) and M1, M3 and M5 (green) are shown in plot A, B and C, respectively. Plot D shows the mean spectra of the pure compounds. These spectra were used for MCR calculations and were SNV corrected and second derivative preprocessed. The peak differences caused by the dominant contribution of stearic acid are arrowed.

molecule was involved in the interaction. A decrease in intensity was seen for the peaks 640, 820, 936, 966, 1210 and $1253 \mathrm{~cm}^{-1}$ whereas peaks $452,720,1014,1586$ and $1614 \mathrm{~cm}^{-1}$ disappeared as is shown in figure 7 Hence, it is suggested that a fraction of MPT became amorphous during the continuous melt granulation process.

Solid state NMR was applied to derive information about the packing arrangement as well as the molecular dynamics of the molecules after melt granulation. No difference in chemical shifts between the granules and the physical mixtures could be detected for stearic acid. For PEO on the other hand, a clear difference in lineshape of the $\mathrm{PEO}$ signal is discovered after melt granulation. Research from Chu et al. revealed the 

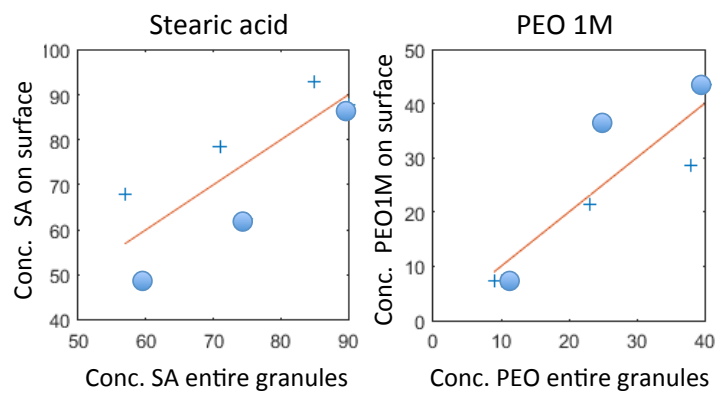

SA/PEO1M granules + SA/PEO1M/MPT granules

Figure 6: Concentrations of SA (left) and $\mathrm{PEO}$ (right) on the surface of the granules of F1, F3 and F5 (circle) and M1, M3 and M5 (cross) ratios calculated using MCR.

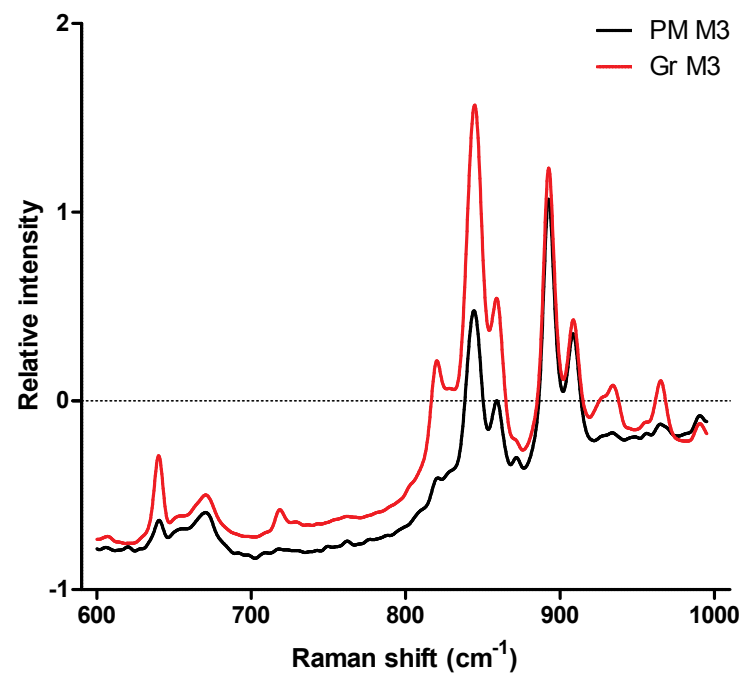

Figure 7: Raman spectrum of physical mixture and granules of blend M3 showing the entire MPT molecule was involved in the interaction with stearic acid.

possibility of using the solid state NMR lineshape and chemical shift to clearly distinguish between amorphous and crystalline $\mathrm{PEO}$ molecules. Whereas a sharp resonance centered at $70 \mathrm{ppm}$ corresponds to highly mobile amorphous $\mathrm{PEO}$, the superimposed broad resonance centered at $72 \mathrm{ppm}$ corresponds to crystalline PEO. The broadening for crystalline $\mathrm{PEO}$ resulted from the interference of decoupling field frequency and frequency of rotational oscillation of PEO helices [? ][? ]. For the drug-free granules, a very sharp peak could be observed at $70 \mathrm{ppm}$ revealing a high fraction of amorphous $\mathrm{PEO}$ (figure 8). Moreover, the intensity 
did not occur in a linear way but showed a large increment in going from $20 \%$ (height treshhold value of 9) to $30 \% \mathrm{PEO}$ (height treshhold value of 26) and stabilized when the $\mathrm{PEO}$ concentration rose to $40 \%$. This observation demonstrated that the fraction amorphous PEO increased between 20 and 30\% PEO. Previous research demonstrated that the degree of crystallinity of $\mathrm{PEO}$ decreased to $43 \%$ in blends containing $\mathrm{PEG}$ 10,000/stearic acid in ratio 1:3 (w/w) [? ]. This revealed that the crystallization of PEO was hindered in $\mathrm{PEO}$ fatty acid blends resulting in a lower degree of polymer crystallinity as compared to the pure PEO. having a crystallinity of $73 \%$. They explained that PEO and fatty acid chains were very close to each other in the blend (due to hydrogen bond formation between both) and therefore the fatty acid impeded the PEG to form well-defined crystals. The chemical shift and lineshape of all NMR signals of stearic acid remained identical for the granules with varying $\mathrm{PEO} /$ fatty acid ratio and, hence, allow to conclude that the solid state of stearic acid is similar in the different drug-free granulates.

Adding 5\% MPT to the granulation process, caused changes in the structural arrangement of the $\mathrm{PEO}$ molecules as could be seen in the broadened and, relatively to the stearic acid signals, decreased intensity of the NMR signals (Figure 8). Addition of MPT to the blend resulted in partial crystallization of the PEO The favored SA MPT interaction took place at the expense of the SA PEO interaction, hence, causing partial phase separation of PEO which subsequently crystallized.

As aforementioned, Pielichowski et al. have studied fatty acid/PEO systems already extensively. They observed that solidification of stearic acid takes place before crystallization of PEO starts. When crystallization of $\mathrm{PEO}$ is initiated, $\mathrm{PEO}$ crystals can only be formed in the limited space between existing fatty acid crystal structures. The limited space caused a high density and, hence, an increased viscosity of the $\mathrm{PEO}$ melt at crystallization temperature facilitating the PEO crystallization [? ][? ]. However, this crystallization process of $\mathrm{PEO}$ is hindered when hydrogen formation between $\mathrm{SA}$ and $\mathrm{PEO}$ took place [? ]. This explains the more amorphous state of the PEO chains in the drug free SA PEO granules. Since addition of MPT hinders these hydrogen bonds, $\mathrm{PEO}$ crystallization can continue resulting in a higher $\mathrm{PEO}$ crystallinity in the SA PEO MPT granules.

Both FTIR and NMR experiments revealed that the hydrogen bond formation between $\mathrm{SA}$ and $\mathrm{PEO}$ is hindered when adding MPT to the blend. Hence, the sustained release properties of the MPT molecules from the SAPEO matrix could not be attributed to hydrogen bond formation between the matrix molecules. 

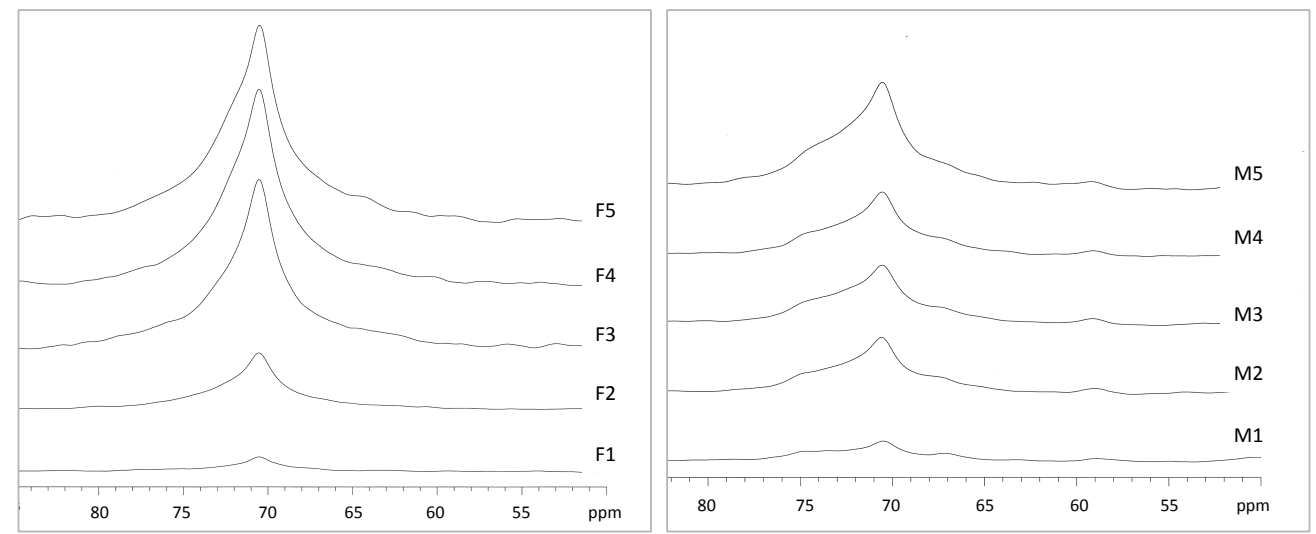

Figure 8: NMR signals of PEO for F1-F5 (left) and M1-M5 (right)

According to Vervaeck et al., the release rate can also depend on the homogeneity of the distribution of PEO throughout the fatty acid matrix. They discovered that the higher molecular weight PEG was less homogeneously distributed throughout the matrix. These pockets with a high PEG concentration affected the porosity of the fatty acid matrix. $\mathrm{PEG}$ molecules leached more easily from these PEG rich pockets and, hence, the MPT release rate was faster [? ]. T1-relaxation measurements were executed in order to study the homogeneity of the distribution of $\mathrm{PEO}$ throughout the stearic acid matrix. The T1H relaxation decay time can provide information regarding the phase morphology in a solid mixture via the process of spin diffusion by which energy is diffused throughout the spin system by successive energy conserving spin flips [? ][? ]. The maximum diffusive path length $L$ can be approximated by the Fickian diffusion equation $\mathrm{L}=(6 . \mathrm{D} . \mathrm{T} 1 \mathrm{H})^{1 / 2}$, with $D$ the spin-diffusion coefficient $\left( \pm 4 \times 10^{-16} \mathrm{~m}^{2} / \mathrm{s}\right)$, and allows to estimate the size of phase separated domains. Phase specific T1H decay times will only be observed if the domain sizes exceed the average diffusive path length. Otherwise a single averaged decay time will be observed. Pure stearic acid has carbon resonances at 15.4, 25.4, 33.1 and $182 \mathrm{ppm}$ and the $\mathrm{T} 1 \mathrm{H}$ decay time measured via these signals is $12.8 \mathrm{~s}$. PEO on the other hand has a carbon resonance around 70-72 ppm and shows a T1H decay time of $4.3 \mathrm{~s}$. A single $\mathrm{T} 1 \mathrm{H}$ decay time for $\mathrm{PEO}$ indicates that the $\mathrm{T} 1 \mathrm{H}$ values of crystalline and amorphous $\mathrm{PEO}$ are averaged out by spin-diffusion, from which it can be concluded that the pure PEO crystals were smaller than $100 \mathrm{~nm}$. For the physical mixtures, the signals of the composing constituents show, as expected, their component characteristic T1H values. T1H relaxation analysis of granules SA/PEO 75/25 (F3) demonstrated significantly decreased $\mathrm{T} 1$ values towards 9.8 and $2.1 \mathrm{~s}$ for $\mathrm{SA}$ and $\mathrm{PEO}$ respectively, in comparison with the physical mixture. This might be due to the hydrogen bond formation between $\mathrm{SA}$ and $\mathrm{PEO}$. Since the $\mathrm{T} 1$ values of stearic acid and $\mathrm{PEO}$ were different, it was evidenced that both components were not ho- 
mogeneously distributed (on a scale of tens to hundreds of $\mathrm{nm}$ ) but phase separated into large domains. The PEO domains appeared to be larger than $35 \mathrm{~nm}$ and the polymer appeared mainly in the amorphous phase as was demonstrated in the NMR spectra (Figure 8 left). The T1H relaxation of granules SAPEOMPT M3 showed, in agreement with the drug free granules, different T1H values for both SA $(10.8 \mathrm{~s})$ and PEO $(2.3 \mathrm{~s})$, demonstrating that also here the components behave as two separate phases. Also here, the PEO domains are larger than $35 \mathrm{~nm}$, but the polymer appeared in the semi-crystalline state according the NMR spectra (Figure 8 right). Moreover, since a single T1H value is found for the semi-crystalline PEO, it can be concluded that the $\mathrm{PEO}$ in the crystals is, due to spin-diffusion, able to relax very efficiently (i.e. with short $\mathrm{T} 1 \mathrm{H}$ value) via the amorphous $\mathrm{PEO}$ chains, indicating that the $\mathrm{PEO}$ crystals have to be smaller than $35 \mathrm{~nm}$. However, the $\mathrm{T} 1 \mathrm{H}$ relaxation value of stearic acid increased by $\pm 1 \mathrm{~s}$ in comparison with the drug-free SA PEO granules. These results confirmed the hypothesis that MPT starts interacting with the fatty acid and, hence, hindering the $\mathrm{SA} / \mathrm{PEO}$ interaction.

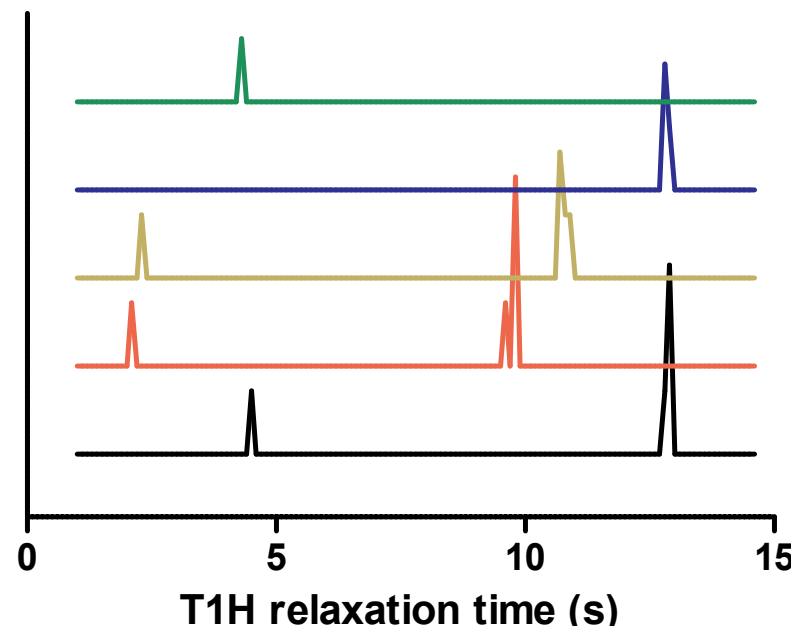

- PM F3 and M3

- Gran F3

- Gran M3

- Stearic acid PEO

Figure 9: The T1 relaxation time distribution of SA, PEO1M, physical mixtures F3 and M3 and corresponding granules.

DSC experiments were performed in order to confirm the findings obtained from the NMR studies. Therefore, the enthalpic value $(\mathrm{J} / \mathrm{g})$ and onset melting temperature were determined of both the physical mixtures and corresponding granules (Table 1). Thermal analysis of the MPT containing physical mixtures and corresponding formulations revealed only one melting endotherm derived from the fatty acid and PEO molecules. Due to dissolution of the MPT crystals in the molten stearic acid during the measurement, no 
MPT melting endotherm could be observed. In the drug-free granules, the enthalpic value was significantly reduced in comparison with the melting enthalpy of the physical mixtures (Figure 10). This difference even increased at higher $\mathrm{PEO}$ fractions. These findings confirmed the results of the NMR experiments that less crystalline material was present after granulation of the drug-free $\mathrm{SA} / \mathrm{PEO}$ blends. Besides the reduction in the enthalpic value of the granules, also the melting onset temperature of the drug-free granules showed a significant decrease in comparison with the physical mixtures. As a result broadening of the melting range did occur. Pielichowski and Flejtuch experienced the same during thermal analysis of fatty acid/PEG blends. The DSC curves also displayed broadening of the temperature range during the second heating in comparison with the phase transitions of pure components, with a temperature shift towards lower values. This was explained by the fact that crystallization was impeded after melting fatty acids and PEG together. If more PEG was present in the blend, the hindered crystallization caused more and more 'defected structures' which are characterized by a lower melting temperature in the second heating [? ][? ]. When MPT was added to the blends before granulation, the onset melting temperature of the granules showed a similar decrease as was seen for the drug-free granules. However, the melting endotherm of the MPT-containing granules exceeded the enthalpic value of the physical mixtures (Figure 10 ). Since this phenomenon was more pronounced at higher $\mathrm{SA}$ concentrations, this is probably due to the interaction between $\mathrm{MPT}$ and $\mathrm{SA}$. An increase in the melting enthalpy of fatty acid was seen earlier by Vervaeck et al. who explained that the excess of energy $(8.38 \mathrm{~J} / \mathrm{g})$ was needed to dissolve the $30 \%$ MPT crystals. Additionally, because stearic acid interacts with the drug, the $\mathrm{SA}$ PEO interaction is hindered. Hence, $\mathrm{PEO}$ crystallizes and the extent of crystallization can exceed the crystallinity of the physical mixtures where the pure PEO had a crystallinity of $73 \%$.

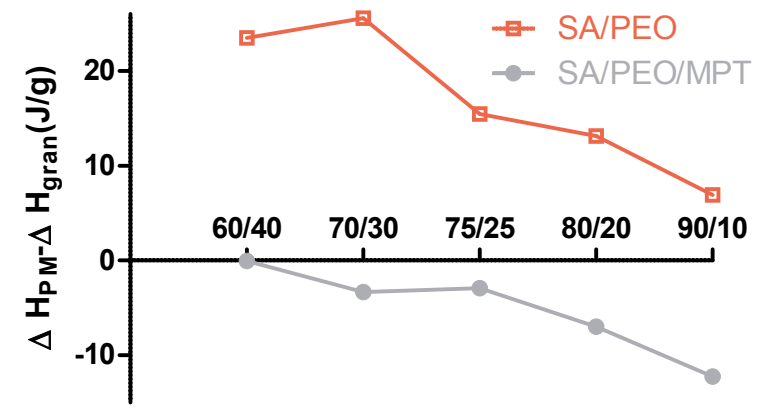

Figure 10: Difference in enthalpic value between physical mixtures and granules for both drug-free $(\mathrm{F} 1-\mathrm{F} 5)$ and MPT containing (M1-M5) blends. 
The solid state of $\mathrm{PEO}$ was also characterized using XRD in order to confirm the previous observations. The X-ray diffraction patterns of the pure compounds, the drug-free SA PEO and SA PEOMPT physical mixtures, and the corresponding granules are shown in Figure 11. MPT showed representative peaks for $2 \theta$ at $10.7^{\circ}, 16.0^{\circ}, 19.6^{\circ}$, and $23.3^{\circ}$. $\mathrm{PEO}$ on the other hand, showed representative peaks for $2 \theta$ at $19.3^{\circ}$, $23.4^{\circ}, 26.3^{\circ}, 27.06^{\circ}, 35.2^{\circ}, 36.2^{\circ}$, and $39.68^{\circ}$. Since the X-ray diffraction patterns of MPT and PEO contain overlapping peaks, only the results from the XRD measurements of the drug-free blends (F1-F5) can be used to evaluate the solid state of $\mathrm{PEO}$ For the drug-free $\mathrm{SA} \mathrm{PEO}$ granules can be seen that the representative $\mathrm{PEO}$ diffraction peaks broadened in comparison with the X-ray diffraction pattern of the drug-free physical mixtures, demonstrating $\mathrm{PEO}$ existed in a more amorphous state and, hence, confirming the NMR and DSC results (Figure 11 left). On the other hand, XRD appears to be very useful to evaluate the crystallinity of the stearic acid chains inside the granules. The X-ray diffraction patterns of the drug-free SA PEO granules showed reduced intensity for the representative peaks of stearic acid. The less structured organization of the fatty acid chains can be due to the interaction between both components. However, after addition of $5 \%$ MPT to the blend, the MPT molecules started competing with $\mathrm{PEO}$ to interact with the stearic acid chains and, hence, the $\mathrm{SA} / \mathrm{PEO}$ interaction is hindered. As a consequence, the stearic acid peaks became more prominent in the diffractogram of the MPT containing granules in comparison with diffractogram of the drug-free granules (Figure 11 right). Since stearic acid plays an important role acting as a barrier impeding the water to penetrate in the $\mathrm{PEO}$ domains, the higher crystallinity of stearic acid reveals that the crystalline structure of the barrier remained intact, which contributes to the slower drug release of MPT from the matrix.
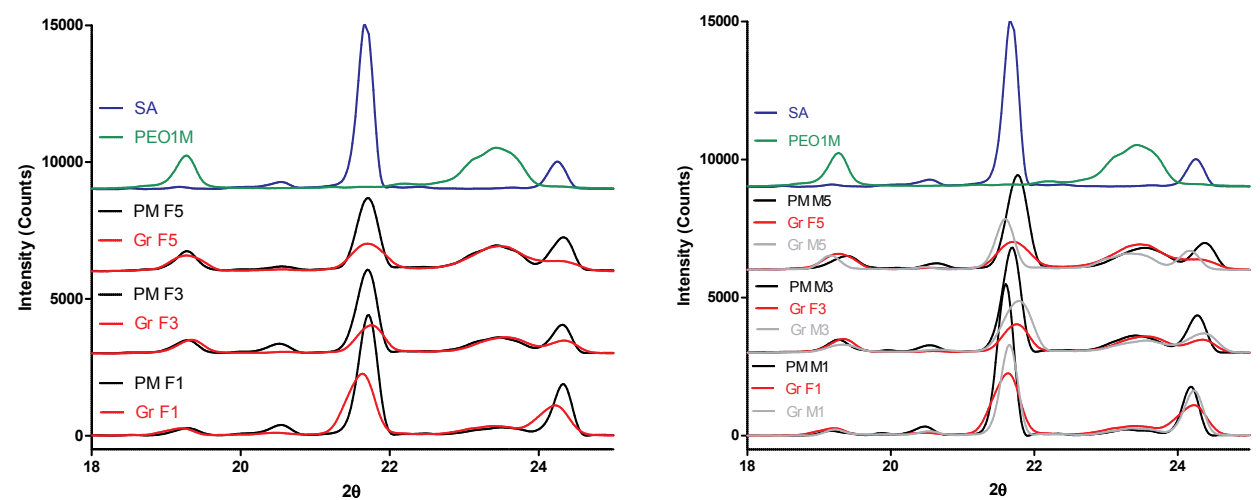

Figure 11: X-ray diffraction patterns of pure $\mathrm{SA}$ (blue) and $\mathrm{PEO}$ (green), with (left) the drug free granules (F1, F3 and F5) (red) in comparison with the corresponding physical mixtures (black) and (right) the M1, M3 and M5 physical mixtures (black) with corresponding granules (grey) in comparison with the drug-free granules (red). 
Additionally, the XRD data of the SA PEOMPT physical mixtures revealed diffraction peaks of MPT for $2 \theta$ at $10.7^{\circ}, 12.04^{\circ}$, and $19.7^{\circ}$, which disappeared/broadened for the corresponding granules. These results revealed that MPT existed in the amorphous form and confirmed the FTIR and Raman results demonstrating that MPT was interacting with stearic acid.

It has to be highlighted that inside the granules, MPT is interacting with the lipophilic phase and, hence, MPT molecules can get released into the dissolution medium via two pathways. On the one hand, they can diffuse to hydrophilic $\mathrm{PEO}$ domains inside the granules from where they can diffuse further to the dissolution medium. On the other hand, the drug particles on the surface of the granules can dissolve immediately into the surrounding dissolution medium. The latter mentioned immediate dissolution results in a burst release [? ]. Since high concentrations of stearic acid correlate with a high burst release, this path has to be the most preferred one for high stearic acid concentrations. This burst effect decreased when the PEO fraction increased. The higher the $\mathrm{PEO}$ concentration was, the lower the amount MPT particles on the surface of the granules was and, hence, the more MPT molecules diffused to the hydrophilic PEO domains, extending their release in the dissolution medium. Apparently, water penetrates into the PEO chains causing swelling of the polymer and, hence, forming a gel-structure. The formation of this gel-phase lengthened the diffusion path of the drug and made diffusion more complicated due to the higher viscosity. Therefore the drug release was extended [? ]. Moreover, water permeability of a polymer in the solid state increases with polymer chain mobility. Since the polymer in the SAPEO MPT granules exists in a highly ordered semi-crystalline state, the water diffusion rate was significantly lower in these crystalline regions than in the amorphous domains. Therefore, the higher crystallinity of the $\mathrm{PEO}$ chains resulted in a slower hydration of PEO chains, slowing down the diffusion process of the MPT molecules into the dissolution medium. This is more pronounced when the granules have a higher $\mathrm{PEO}$ content.

Probably, not only the gel-forming capacity of the high molecular weight $\mathrm{PEO}] \mathrm{s}$ is causing a controlled drug release, but also the lipophilic matrix surrounding the $\mathrm{PEO}$ domains is slowing down the drug release. Since stearic acid is partially covering the $\mathrm{PEO}$ chains, the fatty acid acts as a barrier reducing the surface of the $\mathrm{PEO}$ domains exposed to the outer environment and, hence, controlling water penetration in these domains [? ]. A lower SA PEO ratio reduced the contact surface between PEO and stearic acid (larger PEO domains), contributing to the slower diffusion process of the MPT molecules from the stearic acid matrix into the $\mathrm{PEO}$ domains. 


\section{General conclusion}

This study demonstrated that $\mathrm{SA}$ and $\mathrm{PEO}$ can be used as matrix-formers during twin screw melt granulation and increasing the amount of $\mathrm{PEO}$ extended the release of a highly water soluble drug. The solid state analysis revealed a preferred interaction of the MPT molecules with stearic acid impeding the $\mathrm{PEO}$ to form hydrogen bonds with the stearic acid chains, which was the case in drug-free granules. However, this allowed the $\mathrm{PEO}$ chains to crystallize inside the stearic acid matrix and, hence, elevating the sustained release characteristics of the stearic acid matrix. If water penetrated into the high molecular weight $\mathrm{PEO}$, swelling of the polymer occurred with the formation of a gel-structure making diffusion of the drug more complicated. The increased crystallinity of the polymer hindered the hydration step of the polymer, increasing the release sustaining properties even more. Furthermore, the intact crystalline fatty acid matrix covered the PEO domains inside the granules which made hydration even harder. However, due to the interaction between the MPT and stearic acid molecules, MPT existed in the amorphous form in the fatty acid matrix.

\section{Acknowledgment}

Financial support for this research from the Agency for Innovation by Science and Technology (IWT) is gratefully acknowledged. The research group of P. Adriaensens, and more specifically Gunter Reekmans, is acknowledged for performing the NMR and T1H experiments.

\section{References}

[1] G. M. Walker, G. Andrews, D. Jones, Effect of process parameters on the melt granulation of pharmaceutical powders, Powder Technology 165 (3) (2006) 161-166.

[2] B. Mu, M. R. Thompson, Examining the mechanics of granulation with a hot melt binder in a twin-screw extruder, Chemical Engineering Science 81 (2012) 46-56.

[3] S. Weatherley, B. Mu, M. R. Thompson, P. J. Sheskey, K. P. O'Donnell, Hot-melt granulation in a twin screw extruder: Effects of processing on formulations with caffeine and ibuprofen, Journal of Pharmaceutical Sciences 102 (2013) $4330-$ 4336.

[4] J. P. Lakshman, J. Kowalski, M. Vasanthavada, W.-Q. Tong, Y. M. Joshi, A. T. M. Serajuddin, Application of Melt Granulation Technology to Enhance Tabletting Properties of Poorly Compactible High-Dose Drugs, Journal of pharmaceutical sciences 100 (4) (2010) 1553-1565. 
[5] T. Monteyne, L. Heeze, K. Old??rp, C. Vervaet, J. P. Remon, T. De Beer, Vibrational spectroscopy to support the link between rheology and continuous twin-screw melt granulation on molecular level: A case study, European Journal of Pharmaceutics and Biopharmaceutics 103 (2016) 127-135. doi:10.1016/j.ejpb.2016.03.030.

[6] S. W. Jang, Y. W. Choi, M. J. Kang, Solid Dispersion System for Improved Dissolution of Everolimus Preparation of Solid Dispersion of Everolimus in Gelucire 50/13 using Melt Granulation Technique for Enhanced Drug Release, Bull. Korean Chem. Soc 35 (7) (2014) 1939-1943.

[7] B. Van Melkebeke, B. Vermeulen, C. Vervaet, J. P. Remon, Melt granulation using a twin-screw extruder: A case study, International Journal of Pharmaceutics 326 (February) (2006) 89-93.

[8] B. Evrard, K. Amighi, D. Beten, L. Delattre, a. J. Moës, Influence of melting and rheological properties of fatty binders on the melt granulation process in a high-shear mixer., Drug development and industrial pharmacy 25 (11) (1999) $1177-1184$.

[9] S. Jagdale, S. Ghorpade, D. Bhavsar, B. Kuchekar, Effect of wax on the release pattern of drugs from the sustained release fatty matrix tablet, journal of chemical and pharmaceutical research 2 (2) (2010) 330-338.

[10] J. Kowalski, O. Kalb, Y. M. Joshi, a. T. M. Serajuddin, Application of melt granulation technology to enhance stability of a moisture sensitive immediate-release drug product, International Journal of Pharmaceutics 381 (2009) 56-61.

[11] C. Vervaet, J. P. Remon, Melt granulation, in: D. M. Parikh (Ed.), Handbook of Pharmaceutical Granulation Technology, 3rd Edition, Informa Healthcare, 2010, Ch. 20, pp. 435-448.

[12] M. Savolainen, J. Herder, C. Khoo, K. Lövqvist, C. Dahlqvist, H. Glad, A. M. Juppo, Evaluation of polar lipid-hydrophilic polymer microparticles, International Journal of Pharmaceutics 262 (1-2) (2003) 47-62. doi:10.1016/S0378-5173(03) $00336-3$

[13] E. Verhoeven, T. R. M. De Beer, E. Schacht, G. Van den Mooter, J. P. Remon, C. Vervaet, Influence of polyethylene glycol/polyethylene oxide on the release characteristics of sustained-release ethylcellulose mini-matrices produced by hotmelt extrusion: in vitro and in vivo evaluations, European Journal of Pharmaceutics and Biopharmaceutics 72 (2) (2009) $463-470$.

[14] A. Vervaeck, T. Monteyne, L. Saerens, T. De Beer, J. P. Remon, C. Vervaet, Prilling as manufacturing technique for multiparticulate lipid/PEG fixed-dose combinations, European Journal of Pharmaceutics and Biopharmaceutics 88 (2) (2014) 472-482.

[15] S. B. Tiwari, T. K. Murthy, M. R. Pai, P. R. Mehta, P. B. Chowdary, Controlled release formulation of tramadol hydrochloride using hydrophilic and hydrophobic matrix system., AAPS PharmSciTech 4 (3) (2003) 1-6.

[16] K. Pielichowski, K. Flejtuch, Binary blends of polyethers with fatty acids: A thermal characterization of the phase transitions, Journal of Applied Polymer Science 90 (3) (2003) 861-870. doi:10.1002/app.12775

[17] K. Pielichowski, K. Flejtuch, Differential Scanning Calorimetry Study of Blends of Poly ( ethylene glycol ) with Selected Fatty Acids, Macromolecular Materials and Engineering 288 (2003) 259-264.

[18] K. Pielichowski, K. Flejtuch, Recent developments in polymeric phase change materials for energy storage: poly(ethylene oxide)/stearic acid blends, Polymers for Advanced Technologies 16 (2-3) (2005) 127-132.

[19] K. Pielichowski, K. Flejtuch, Thermal properties of poly(ethylene oxide)/lauric acid blends: A SSA-DSC study, Thermochimica Acta 442 (2006) 18-24.

[20] K. Pielichowska, S. Głowinkowski, J. Lekki, D. Biniaś, K. Pielichowski, J. Jenczyk, PEO/fatty acid blends for thermal energy storage materials. Structural/morphological features and hydrogen interactions, European Polymer Journal 44 (2008) 3344-3360. 
[21] K. Pielichowska, K. Pielichowski, Kinetics of Isothermal and Nonisothermal Crystallization of Poly(ethylene oxide) (PEO) in PEO/Fatty Acid Blends, Journal of Macromolecular Science, Part B 50 (9) (2011) 1714-1738.

[22] J. Jaumot, A. de Juan, R. Tauler, MCR-ALS GUI 2.0: New features and applications, Chemometrics and Intelligent Laboratory Systems 140 (2015) 1-12.

[23] A. Vervaeck, L. Saerens, B. G. De Geest, T. De Beer, R. Carleer, P. Adriaensens, J. P. Remon, C. Vervaet, Prilling of fatty acids as a continuous process for the development of controlled release multiparticulate dosage forms, European Journal of Pharmaceutics and Biopharmaceutics 85 (3 PART A) (2013) 587-596.

[24] P. P. Chu, H. Wu, Solid state NMR studies of hydrogen bonding network formation of novolac type phenolic resin and poly ( ethylene oxide ) blend, Polymer 41 (2000) 101-109.

[25] G. Reiter, G. R. Strobl, Progress in Understanding of Polymer Crystallization, Lecture Notes in Physics, Springer Berlin Heidelberg, 2007.

[26] P. Adriaensens, L. Storme, R. Carleer, J. Gelan, Comparative Morphological Study of Poly ( dioxolane )/ Poly ( methyl methacrylate ) Segmented Networks and Blends by 13 C Solid-State NMR and Thermal Analysis, Macromolecules (2002) 3965-3970.

[27] A. Duki, R. Mens, P. Adriaensens, P. Foreman, J. Gelan, J. P. Remon, C. Vervaet, Development of starch-based pellets via extrusion/spheronisation, European Journal of Pharmaceutics and Biopharmaceutics 66 (1) (2007) $83-94$.

[28] L. Maggi, L. Segale, M. L. Torre, E. Ochoa Machiste, U. Conte, Dissolution behaviour of hydrophilic matrix tablets containing two different polyethylene oxides (PEOs) for the controlled release of a water-soluble drug. Dimensionality study, Biomaterials 23 (4) (2002) 1113-1119.

[29] L. Maggi, R. Bruni, U. Conte, High molecular weight polyethylene oxides (PEOs) as an alternative to HPMC in controlled release dosage forms., International journal of pharmaceutics 195 (1-2) (2000) 229-38. 